\title{
Prospects for the Prevention of Premature Aging With Plant Antioxidants
}

\author{
Praskova Yulia A. ${ }^{1, *}$ Frolova Nina A. ${ }^{1}$ Kiseleva Tatiana F. ${ }^{2}$ \\ Matyushchenko Victoria $\mathrm{S} .{ }^{3}$ Reznichenko Irina $\mathrm{Yu} .{ }^{2}$
}

\author{
${ }^{1}$ Amur State University, Blagoveshchensk, Russia \\ ${ }^{2}$ Kemerovo State University, Kemerovo, Russia \\ ${ }^{3}$ Amur State Medical Academy, Blagoveshchensk, Russia \\ *Corresponding author. Email: stennna09@ramber.ru
}

\begin{abstract}
Insenescence has intrigued and concerned scientists from the very beginning of civilization. It is a process that can be broadly described as changes that occur during the life cycle of an organism that gradually increases the likelihood of their death. The changes that lead to a gradual increase in the likelihood of mortality span all aspects of biology, from molecular mechanisms to physiological systems of the whole body. Moreover, there is no doubt that aging is externally modulated by lifestyle and diet, while the rate of aging appears to be determined by a wide variety of genetic mutations. In this regard, the prevention of aging is especially relevant, using biologically active substances obtained from wild-growing raw materials with an acceptable safety profile. Resveratrol obtained from Amur grapes can be safely attributed to such substances. The scientific article presents the results of a study of resveratrol as a means of preventing the aging process in animals. The article analyzed the dynamics of changes in lipid peroxidation and peroxide resistance of erythrocytes under conditions of acute stress. As a result of the conducted studies, it was found that the effect of stress contributes to an increase in the degree of hemolysis of erythrocytes, as well as an increase in the content of liver lipid peroxidation products. However, the introduction of resveratrol into the diet of the studied animals contributed to a decrease in lipid peroxidation in the liver of animals and an increase in the resistance of erythrocytes to the action of peroxide compounds, which indicates a slowdown in the aging rate.
\end{abstract}

Keywords: aging, nutrition, resveratrol, Amur grapes, preventive efficacy

disease occurs and how each contributes to mortality, there remains a strong basis for considering diseases in their totality.

Since the middle of the 19th century, the demographic situation in the world has had a negative birth rate dynamics with a low mortality rate $[1,2]$. It became obvious that population growth would gradually slow down. Already in the 20th century, scientists around the world noted an intensive process of "demographic aging". Studies of this process made it possible to establish that this phenomenon is not temporary.

In 2018, in the Russian Federation, the number of old people aged 70 and over was $9.2 \%$ of the total population.

The simplest measure of aging is chronological age because the mortality rate increases exponentially with age, in accordance with Gompertz's law of mortality. Despite this, the mortality rate is a characteristic of the population and its application to individuals is very limited. This is due to the fact that people at the same age differ from each other not only because of differences in their genetic profile, but also because of how the external environment, incl. and nutrition influence many biological pathways for controlling and repairing damage. This variability in damage potential and control leads to 
variable changes in physiological functions, loss of homeostatic regulation, reduction in homeodynamic space, flexibility, and vulnerability to stressors.

The aging process begins before birth with epigenetic changes that affect gene regulation. In addition to fundamental changes in organ structure, it is also increasingly shown that epigenetic mechanisms, which are prone to the presence or absence of certain nutrients during critical growth periods, establish long-term patterns of gene expression.

A persistent topic of research by various scientists over the past two decades is that aging is not a passive accumulation of cellular damage, but active regulation at the level of the whole organism.

The mechanism is modulated by an internal calculation based on external signals, so that damage can accumulate at a variable rate, without the body having to follow the planned program.

Despite the great individual variability in the manifestation of aging, there is a universal characteristic the number of health problems increases with age. Taking this into account, prognostically grounded recommendations for the primary prevention of premature aging can be formulated, including the general principles of health preservation [2].

Health preservation is the basis of disease prevention. The goal of health preservation is to achieve the highest possible level of health, functioning and adaptation of both healthy people and people with physical and mental pathology, social disadvantage.

Health is a key value for Russians, especially for people of the older generation, determining the factor of working capacity. Loss of health for the elderly is a dangerous factor in increasing the cost of medical care and decreasing the quality and diet of food, decreasing vitality, and falling living standards.

Our nutritional health depends not only on essential vitamins and minerals, but also on the optimal balance of macronutrients that nourish our bodies, but also on other bioactive compounds in our food, commonly called phytonutrients.

Recent analyzes of national studies of food consumption in Russia and around the world show that, despite dietary recommendations that emphasize the importance of increasing the consumption of naturally occurring nutrients found in fruits and vegetables, consumption worldwide is lower than recommended.

Optimizing the consumption of certain foods and / or their biologically active components is a reasonable and cost-effective strategy for disease prevention. However, determining the "ideal" diet is difficult for a number of reasons, including the difficulty of determining the amount of a particular food or nutrient needed to achieve a desired response, and the multiple interactions of some nutrients with other nutrients.

While there is not enough information to formulate the "ideal diet to stop aging," there is enough knowledge to warrant a call for future nutritional health research. In addition, it is becoming increasingly apparent that people do not respond in the same way to the foods they consume. Therefore, as we begin to better understand the critical role that multiple food components play in regulating cellular events and how they are influenced by genetic and epigenetic events, cultural and lifestyle differences, and our individual physical and social environment, the more we recognize the need to an individual approach to optimizing our nutrition. As bioactive compounds, the number of which is growing, have yet to be fully understood, the importance of food in general, and not just the nutrients it provides, is again becoming a primary issue.

Of the many concepts of aging that exist at the present time, the main ones include elevation, apoptosis, free radical. They are all somehow interconnected.

Among the famous scientists who studied the biology of the aging of the body, Academician of the USSR Academy of Medical Sciences Davydovsky I.V., who in his unique monograph "Gerontology" described the mechanism of the biology of aging in detail, systemic organ gerontology and geriatrics [5]. In his scientific works Davydovsky I. V. divided the concepts of "old age" and "aging", noting that the determination of old age is integral and covers the entire organism. At the same time, academician Davydovsky noted that in gerontology there is a synthesis of self-determination and external causal determination, which, to a certain extent, allows one to influence the course of aging. It was Davydovsky I.V., with his research, laid the foundation for gerontology, and proposed ways to prevent aging, which have received intensive development nowadays [5, 7].

Currently, extensive information has accumulated that the rate and degree of development of diseases happen due to the impact of a number of stress factors: the environment, lifestyle, nutrition, as well as the interaction of these factors with each other. As a consequence, there is a decrease in life span with subsequent premature death due to the activation of free radical oxidation reactions $[8,9,12]$.

Aging is the process of decreasing the overall vitality of the body with age. The aging processes of the human body are launched individually, long before the onset of the formal ("passport") age of old age. According to Davydovsky I.V. it is associated with morphological changes in various organs $[5,13]$.

The main reason for aging for different organs is the law of entropy in individual systems:

- body pollution;

- loss of non-renewable elements of the body;

- accumulation of damage and deformation;

- transformation of regulation processes.

It is necessary to build the correction of age-related changes in a comprehensive manner, harmonized throughout life, taking into account the peculiarities. 
The applied methodological approaches to the prevention of aging should be based on the ratio "safety / effectiveness".

Based on this, biologically active substances (antioxidants) contained in wild berry raw materials are of great interest [11].

Such substances from the group of polyphenols can be safely attributed to resveratrol, obtained from the fruits and seeds of the Amur grape. Empirical use of resveratrol testifies to its positive effect in normalizing cell metabolism, enhancing oxygen transport, improving blood rheological parameters, strengthening the vascular wall, as well as antiviral and antibacterial effect.

Resveratrol was first isolated from dark grape varieties. However, various scientists have proven the presence of resveratrol in 70 different plants (mulberries, peanuts, cocoa beans, pine bark, etc.).

Free radical oxidation aggravates the course of various diseases, thereby accelerating the aging process of the body. The antioxidant activity of resveratrol is closely related to its immunomodulatory properties.

The purpose of this study was to evaluate the effectiveness of using resveratrol obtained from the fruits and seeds of Amur grapes in the complex prevention of premature aging.

\section{METHODS AND MATERIALS}

Experimental studies were carried out on rats of two age categories: 150 old rats and 150 young rats. The average weight of animals in the age category was $220 \mathrm{~g}$, in the young category $85 \mathrm{~g}$.

All animals were kept in natural light under standard vivarium conditions. The rats were fed synchronized with free access to water. The experiment lasted 21 days.

Each age category of animals was divided into 6 groups:

Group 1 - intact animals.

Group 2 - animals on a standard diet, stressed.

Group 3 - animals whose diet included resveratrol, obtained from Amur grapes, in a daily dose of $0.5 \mathrm{mg}$.

Group 4 - animals whose diet included resveratrol, obtained from Amur grapes, in a daily dose of $1.0 \mathrm{mg}$.

Group 5 - animals whose diet included resveratrol, obtained from Amur grapes, in a daily dose of $0.5 \mathrm{mg}$, subjected to stress.

Group 6 - animals, whose diet included resveratrol obtained from Amur grapes, in a daily dose of $1.0 \mathrm{mg}$, subjected to stress.

The animals were placed in a plastic chamber at a temperature of $20-22{ }^{\circ} \mathrm{C}$; their movement was limited, thereby causing acute stress.

At the end of the experiment, the animals were decapitated. The studies were carried out at the same time after slaughter simultaneously in all groups.

Studies on changes in liver lipid peroxidation (LPO) were determined by the method of Stalnaya I.D. [14]

The results were processed statistically using the arithmetic mean, its error, and the significance of the difference between the means of the compared groups using the Student's t test.

\section{RESULTS}

The obtained results of experimental studies made it possible to establish (Fig. 1,2) that the introduction of resveratrol into the diet of animals significantly reduces the level of malondialdehyde (MDA) in the liver of old rats, incl. initial spontaneous and ascorbine-dependent. An increase in the concentration of lipid peroxidation products in the liver of animals of the old age group is also observed, with a significant decrease in the level of antioxidants. The decrease in the initial level of liver lipid peroxidation in the category of old animals was $19.63 \%$ in the fifth group, $22.4 \%$ in the sixth group compared with the animals of the second group, which is statistically significant when resveratrol is introduced into the diet. However, no significant differences were found with different doses of resveratrol.

In young rats, in comparison with the category of old animals, the level of liver lipid peroxidation was significantly lower, which indicates a clearer effective effect of resveratrol on intact young animals.

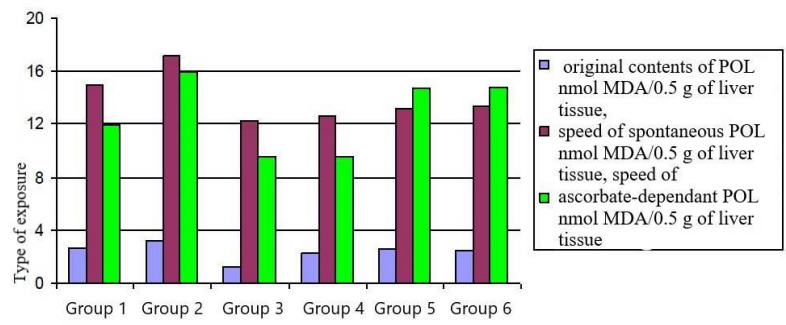

Figure 1 Dynamics of the effect of resveratrol and stress on the lipid peroxidation rate in the liver of old rats

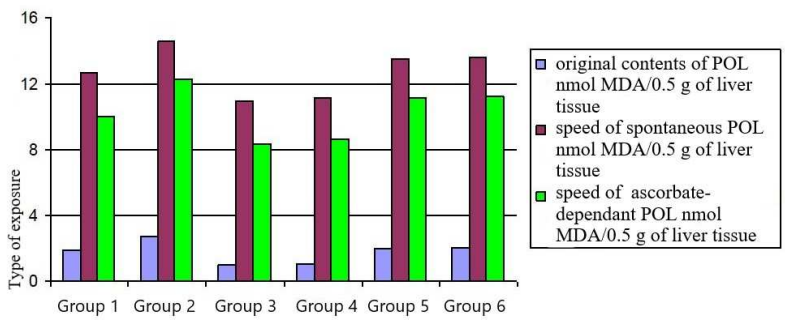

Figure 2 Dynamics of the effect of resveratrol and stress on the LPO rate in the liver of young rats

As a result of the experiments, a pronounced stressprotective effect was established in animals whose diet included resveratrol obtained from Amur grapes (without significant differences in the dose of administration), which was expressed in a significant approximation of all lipid peroxidation parameters to the control level. Agerelated differences in the stress-protective effect of 
resveratrol at various concentrations in the diet of animals were not found.

The main sensitive indicator of the state of the system "oxidants-antioxidants" is peroxide hemolysis of erythrocytes $[3,4,12]$. In this regard, simultaneously with the determination of LPO in the liver of rats, studies were carried out to determine the peroxide hemolysis of erythrocytes of animals of two age categories. During the experiment, a significant peroxide resistance of erythrocytes was noted in young animals of the control group compared with old animals of a similar group. When introduced into the diet of resveratrol in different doses, it helped to reduce the hemolysis of erythrocytes in animals of all age groups. Analyzing the effect of stress on changes in erythrocytes, their less noticeable resistance to destruction was noted. The stress-protective effect of resveratrol in older animals was less pronounced.

\section{CONCLUSION}

Over the past decades, in many countries of the world, including Russia, there has been a process of population aging. This problem has traditionally been considered only within the framework of ethical and social problems. But over the past century, society has realized that the aging process needs to be investigated in another aspect: as a special physiological mechanism of the body that has a certain evolutionary significance. Changes in the age structure of the population that have been taking place for more than a decade - a decrease in the proportion of children in the total population and an increase in the proportion of older people - have an ever-increasing impact not only on demographic dynamics, but also on socio-economic development.

In many countries of the world, the proportion of older people is growing rapidly, which forces a new study of the problem of aging.

Aging is the most difficult problem in medicine and biology. The aging process is a gradual tissue involution and disruption of body functions. Symptoms of old age appear at the end of the reproductive period and become more intense as we age.

It is known that the species limit of human life expectancy is approximately 30-40 \% higher than the average life span. This is due to the effect on the body of various unfavorable factors that lead to a change in the expression and structure of genes, which is accompanied by a violation of protein synthesis and a decrease in body functions.

Thus, the studies carried out have shown that in animals of the old age category, free radical processes in the liver tissue increase and proceed more intensively in comparison with young rats. The introduction of resveratrol into the diet of animals helps to reduce the level of lipid peroxidation, regardless of the dose. This was most clearly observed in young rats. In animals of the old age category, immobilization stress led to an intensification of free radical processes more effectively than in animals of the young age category. In animals of all age categories, a pronounced stess-protective effect was noted when resveratrol, obtained from Amur grapes, was introduced into the diet, regardless of the dose. Under conditions of acute stress in the blood of young rats, erythrocytes were resistant to their destruction. Old rats, which diet included resveratrol obtained from Amur grapes, have shown a less pronounced stress-protective effect.

During the aging process, the strength of cell membranes decreases due to the activation of free radical oxidation [15]. Antioxidant protection and the intensity of reactions decrease with age, even though free radicals are generated. An increase in oxidative stress in cell membranes occurs due to the products of free radical oxidation. The introduction of resveratrol into the diet helps to inhibit free radical processes. When using resveratrol obtained from Amur grapes, no side effects were observed in animals, which correspond to the principles of modern gerontology and allows us to recommend it for the prevention of aging processes.

\section{REFERENCES}

[1] V.N. Anisimov, M.V. Soloviev, The evolution of concepts in gerontology, Doctor, St. Petersburg, 1999, 130 p.

[2] A.Y. Baranovsky, O.B. Protopopov, O. Khurtsilava, Gerontologia, Advances in gerontol. 2 (2012) 205-216.

[3] X.Z. Brainin, The Method of measurement of antioxidant aktivnosti in foods, Supplements and vitamins method potentiometry, MIM 02.005-06, Publ. house of the Ural state Univer. of Econ., Ekaterinburg, 2006, p. 48.

[4] State Pharmacopoeia of the USSR X1, Moscow, 2007, $720 \mathrm{p}$

[5] Davydovsky, I.V. Gerontology, Medicine, Moscow, 1996, $300 \mathrm{p}$

[6] The customer at the pharmacy: the subtleties of communication with older people, Pharmac. J. 5 (2010). Retrieved from: http://www.pharmvestnik.ru/ publs/staryj-arxiv-gazety/19255.html\#.Uf4B_bbJ0fQ. Date of access: 27.07.2013.

[7] C.K. Lavrenov, G.V. Lavrenova, Complete encyclopedia of medicinal plants, vol. 1, St. Petersburg, 1999, $735 \mathrm{p}$.

[8] A.S. Saratikov, E.A. Krasnov,Rhodiola rosea (Golden root), Monograph, Publ. house of Tomsk Univer, Tomsk, 2004, 292 p.

[9] G.L. Safarova, Demography of aging: current status and research priorities, Advances in gerontol. 1 (2009) 49-59.

[10] Social work with older people abroad, Psychological center the Art of living. Retrieved from: http://www.psyvita.ru/mudrost/46. Date of access: 31.07.2013. 
[11] V.X. Khavinson, V.N. Anisimov, 35 years research experience peptide regulation of aging, Success of gerontol. 1 (2009) 11-24.

[12] V.X. Khavinson, V.V. Malinin, Peptide regulation of genetic stability during aging, Med. Acad. J. 6(1) (2006) 139-143.
[13] C. Borek, Antioxidant health effects of aged garlic extract. J. Nutr. 131 (2001) 1010-1015.

[14] A.D. Stalnaya, L.A. Romanov, Modern methods in biochemistry, Medicine, Moscow, 1997, 352 p. 